95\% CI: 0.64-0.95), and retinopathy (HR: 0.82, 95\% CI: 0.69-0.98) were all significantly lower among patients in the bundled care model. Too few incident amputation cases (11 for Bundle, and 6 for Non-Bundle) limit the power to detect significant hazard difference for amputation (HR: 1.32, 95\% CI: 0.45-3.85). Above findings are based on an observational design and the population is limited to those enrolled in a health plan in central and northeastern Pennsylvania. Discussion: A primary care all-or-none bundle of measures (Bundle) for management of patients with diabetes may reduce the risk of microvascular and macrovascular events over time. The effectiveness of the Bundle management could be observed in as early as two years.

Keywords: Diabetes; All-or-one Bundle of Measures doi:10.3121/cmr.2012.1100.ca6-03

CA6-05:

Improved Glycemic Control from Diabetes Self-management Education is Not Sustained

JoAnn Sperl-Hillen'; Sarah Beaton²; Omar Fernandes'; Ann Von Worley²; Gabriela Vazquez-Benitez'; Ann Hanson'; Jodi Lavin-Tompkins'; William Parsons $^{2}$; Vic Spain ${ }^{3}$

${ }^{1}$ HealthPartners; ${ }^{2}$ Lovelace Health System; ${ }^{3}$ Merck and Co, Inc.

Background/Aims: For patients with type 2 diabetes of long duration, we evaluated whether improved short-term outcomes obtained through diabetes education were sustained. Methods: 623 adults with glycosylated hemoglobin (A1c) greater or equal to $7 \%$ were randomized to individual education (IE), group education (GE) using Conversation Maps, or usual care (UC). Alc, Problem Areas in Diabetes (PAID), Diabetes Self-Efficacy (DES), Recommended Food Score (RFS), Physical Activity (PA), and medication intensification (an increase in number of medication classes or insulin start) were evaluated at baseline and at approximately 6 month intervals for the following year using linear mixed models. Results: Compared to UC, IE resulted in sustained improved DES and PAID scores in the long-term (DES, $+0.11, \mathrm{p}=.03$ and PAID, -2.94, $\mathrm{p}=.04$ ), but not significant RFS or PA longterm change. IE resulted in a short-term A1c reduction of .25\% $(\mathrm{p}=.03)$ and odds ratio (OR) of 1.83 (1.05-3.17) for achieving an Alc $<7 \%$ compared with UC, but significant effects were not observed after 6 additional followup months. No differences were observed between GE and UC for short-term and long-term DES, PAID, RFS, PA, or A1c. In patients with pharmacy claims data $(\mathrm{n}=488)$, odds ratios of medication intensification comparing IE to UC were short-term $0.83(0.44-1.57)$ and long-term $0.79(0.43-1.47)$; comparing or GE to UC were short-term 1.22(0.66-2.26) and long-term 0.92(0.50-1.68). Discussion: In patients with suboptimal glycemic control, improved measures of self-efficacy and lower diabetes distress observed with IE in the short-term were sustained long-term. However, short- term improvements in glucose control, nutrition, and physical activity were not sustained. Educational interventions did not result in higher medication intensification rates. Results are consistent with most behavior change theories, and suggest greater attention to maintenance and relapse, possibly from continued ongoing support from educators, may be needed to sustain improvements in healthy behaviors and glucose control.

Keywords: Diabetes; Patient Education

doi:10.3121/cmr.2012.1100.ca6-05

\section{Health Informatics}

PS1-37:

Regional Epidemiologic Assessment of Prevalent Periodontitis Using an Integrated Electronic Health Record System

Amit Acharya ${ }^{1}$; Jeffrey VanWormer ${ }^{1}$; Stephen Waring ${ }^{2}$; Aaron Miller ${ }^{1}$; Jay Fuehrer'; Gregory Nycz ${ }^{1}$

\section{${ }^{1}$ Marshfield Clinic; ${ }^{2}$ Essentia Health}

Background/Aims: The purpose of this study is to describe an oral health surveillance platform that queries a clinical/administrative data warehouse to estimate the regional prevalence of periodontitis. Methods: A cross-sectional analysis was performed using electronic health records (EHR) data collected 01/01/2006-12/31/2010 from the Marshfield Epidemiologic Study Area. The sample was drawn from the rural Ladysmith, WI area $(\sim 13,000$ people), which is primarily served by Marshfield Clinic. Eligibility criteria were: (1) home address in the $54848,54526,54731,54530,54819$, or 54563 zip codes, (2) age 25-64, and (3) =1 Marshfield Dental Clinic comprehensive exam. The outcome of interest was prevalent periodontitis and was established using two independent. The first method utilized an algorithm that considered clinical attachment loss and probe depth to identify moderate and severe periodontitis cases, consistent with established national definitions. The second method was based on standardized current dental terminology (CDT) codes related to periodontal treatment procedures. Prevalence estimates were direct age-standardized to the year 2000 U.S. census estimates. Results: Of the 7,676 eligible individuals in the region, 2,056 (27\%) had $=1$ comprehensive exam and thus were included in the analytical sample. Based on the AAP/ CDC method, the prevalence of moderate periodontitis was 373/1,000 males, and 285/1,000 females. The prevalence of severe periodontitis was 33/1,000 males, and 24/1,000 females. The age-standardized prevalence of moderate or severe periodontitis (combined) was 407/1,000 males, and 308/1,000 females. Combined moderate/severe prevalence estimates were somewhat lower using the CDT code method, where the prevalence of moderate/severe periodontitis was $348 / 1,000$ males, and 269/1,000 females. There was a general pattern of increased prevalence and severity of periodontitis with increased age. Discussion: The prevalence of periodontitis in this area was consistent with national estimates, but the tested methodology highlighted the need to address some potential some forms of sample selection bias in future EHR-based periodontitis research. As EHR use increases in dental systems around the country, the

Keywords: Integrated Electronic Health Record; Prevalent Periodontitis; Health Informatics

doi:10.3121/cmr.2012.1100.ps1-37

PS1-38:

A Simulation Study to Evaluate the Feasibility of Conducting Active Surveillance to Detect Clusters of Allograft Infection

Lingling $\mathrm{Li}^{1}$; Ken Kleinman ${ }^{1}$; Fang Zhang ${ }^{1}$; Michael Klompas ${ }^{1}$

${ }^{1}$ Harvard Pilgrim Health Care Institute, Harvard Medical School

Background/Aims: Infection following implantation of an orthopedic allograft can be devastating. Infections caused by contaminated allograft tissues are believed to be rare, but clusters - groups of infections with a common cause - have been discovered and reported repeatedly. Discovering clusters is challenging, however, because most orthopedic infections are attributed to surgical infection rather than allograft contamination. Statistical cluster detection tools may facilitate recognition of allograft contamination patterns. It is not known, however, whether the combination of number of surgeries, suppliers, hospitals, and infection rates in the US would offer sufficient power to detect allograft contamination signals, even if the FDA were to establish a national surveillance system. We therefore sought to model the feasibility of detecting clusters through active surveillance of allograft infection. Methods: We simulate data that might be collected through such a surveillance system. We consider the ability of scan statistics to detect synthetic clusters of infection that reflect either manufacturing contamination or hospital error. We evaluate the power of scan statistics to detect infection clusters under a range of scenarios with varying factors including number of allograft implantations, baseline manufacturing contamination rate, baseline hospital error rate, elevated manufacturing contamination rate, elevated hospital error rate, and cluster size. The simulation settings are specified according to published statistics and with inputs from experts with subject matter knowledge in an attempt to mimic real-life settings. Results: Power increases with the number of allograft implantations, the cluster size, the relative contamination rate between the cluster and the baseline, and the relative surgical error rate between the cluster and the baseline. Power ranges from 0.1 under very conservative assumptions to nearly 1.0 with the greatest prevalence and contamination rates, with realistic scenarios resulting in power $>0.8$. For example, there is power of 0.80 to detect a medium supplier $(2,000$ tissues per year) with a contamination relative risk of 8 compared to other suppliers, presuming $60 \mathrm{~K}$ procedures per year, 20 suppliers, 1025 hospitals, $0.1 \%$ baseline contamination rate, and $0.1 \%$ baseline hospital error rate. Discussion: An active allograft 